\title{
Preface: EmergeNET4: engineering emergence
}

\author{
Paul S. Andrews $\cdot$ Susan Stepney
}

Published online: 10 April 2012

(C) Springer Science+Business Media B.V. 2012

EmergeNET is a UK EPSRC-funded network (EP/E0628 14/1) to promote interest in complex systems and emergence by bringing together established researchers, those new to the area, and the public.

In 2010, EmergeNET sponsored its fourth workshop, in York, on the theme of Engineering Emergence in complex systems. Complex systems are characterised by low-level components that communicate and interact with and within an environment, resulting in the emergence of high-level system behaviours and properties. Complex systems are often designed to help understand emergence or to exploit emergent behaviours to help solve problems in the real world. Emergent behaviours are by definition a function of the overall system rather than of any individual component, which makes the engineering of these behaviours a nontrivial activity.

After the workshop, an open call for a special issue of Natural Computing on the topic of Engineering Emergence was launched. Of the papers submitted to this call, five were selected for publication after review. The papers presented here cover a broad range of issues to do with engineering emergence, including design principles, simulation architectures, and software and hardware applications.

P. S. Andrews · S. Stepney ( $\varangle)$

Department of Computer Science, University of York, York, UK

e-mail: susan.stepney@ york.ac.uk

P. S. Andrews

e-mail: paul.andrews@york.ac.uk

\section{Contributions}

In the first paper ("Degeneracy and Networked Buffering: principles for supporting emergent evolvability in agile manufacturing systems") Frei and Whitacre discuss a particular design principle applied at the component level leading to engineered emergent evolvability. They discuss biologically-inspired role of degeneracy (multi-functional components with partially overlapping functions), and networked buffering, and their relationship to the emergence of evolvability. They apply their ideas to an example of engineering robust manufacturing systems, and argue that the approach enhances ability to access novel design options.

In the next paper ("Evolutionary Mechanics: new engineering principles for the emergence of flexibility in a dynamic and uncertain world") Whitacre et al. investigate the role of degeneracy in more detail, in relation to an engineered system's robustness to environmental change. They illustrate this through a validating application of scheduling a simulated fleet of transportation vehicles, where degeneracy arises in that different vehicle types can carry out some of the same tasks.

In the third paper ("To Boldly Go: an occam- $\pi$ mission to engineer emergence") Welch et al. describe a massively parallel implementation architecture, and use it to experiment with a range of "functional" emergent properties (as opposed to the more commonly investigated "non-functional" emergent properties of robustness, flexibility, and so on). The approach is illustrated through a sophisticated boids simulation. The authors argue that, since we have engineered only the individual components, not the emergent properties, we need to use a new approach to help design the desired emergent properties: the scientific method. 
In the fourth paper ("Programming and Evolving Physical Self-Assembling Systems in Three Dimensions") Bhalla et al. use a combination of evolutionary algorithms, hand design, and rapid prototyping to engineer the components of an emergent self-assembling 3D system. This is emergent engineering applied to a physical system, as opposed to a software system or a simulation.

In the fifth paper ("The Gestalt Heuristic: emerging abstraction to improve combinatorial search") Philemotte and Bersini describe a novel metaheuristic, the Gestalt algorithm, inspired by emergent processes in cognition and perception. Here we see an example of an engineered emergent property that provides a better algorithm.

It is early days in the subject of emergent engineering, and these papers provide some of the first steps in elucidating design principles and approaches, architectures, applications, and analyses. We thank all the authors for their contributions. 